\title{
Erratum to: CX3CR1 deficiency accelerates the development of retinopathy in a rodent model of type 1 diabetes
}

\author{
Eleni Beli $^{1}$ - James M. Dominguez II ${ }^{1,2}$ • Ping Hu ${ }^{1}$. Jeffrey S. Thinschmidt ${ }^{2}$. \\ Sergio Caballero ${ }^{2}$ - Sergio Li Calzi ${ }^{1}$. Defang Luo ${ }^{2}$ - Sumathi Shanmugam ${ }^{4}$. \\ Tatiana E. Salazar $^{1,2}$ - Yaqian Duan ${ }^{1} \cdot$ Michael E. Boulton ${ }^{1} \cdot$ Susanna Mohr $^{3}$. \\ Steven F. Abcouwer ${ }^{4}$. Daniel R. Saban ${ }^{5}$ Jeffrey K. Harrison ${ }^{2}$. Maria B. Grant ${ }^{1,2}$
}

Published online: 18 April 2017

(C) Springer-Verlag Berlin Heidelberg 2017

Erratum to: J Mol Med (2016) 94:1255-1265

DOI 10.1007/s00109-016-1433-0

The original version of this article unfortunately contains a mistake in Fig. 1a; the wrong figure panels were used.

The correct Fig. 1a is shown in this paper.

The online version of the original article can be found at http:/dx.doi.org/ 10.1007/s00109-016-1433-0

Maria B. Grant

mabgrant@iupui.edu

1 Eugene and Marilyn Glick Eye Institute, Department of Ophthalmology, Indiana University School of Medicine, Indianapolis, IN, USA

2 Department of Pharmacology and Therapeutics, University of Florida, Gainesville, FL, USA

3 Department of Physiology, Michigan State University, East Lancing, MI, USA

4 Department of Ophthalmology and Visual Sciences, University of Michigan, Kellogg Eye Center, Ann Arbor, MI, USA

5 Department of Ophthalmology, Duke University School of Medicine, Durham, NC, USA 
Fig. 1 Vascular pathology in the retina. a Representative images of trypsin-digested retinas for acellular capillaries (arrowheads) in nondiabetic Veh-WT, Veh-Het, and Veh-KO mice and in diabetic STZ-WT, STZ-Het, and STZ-KO mice
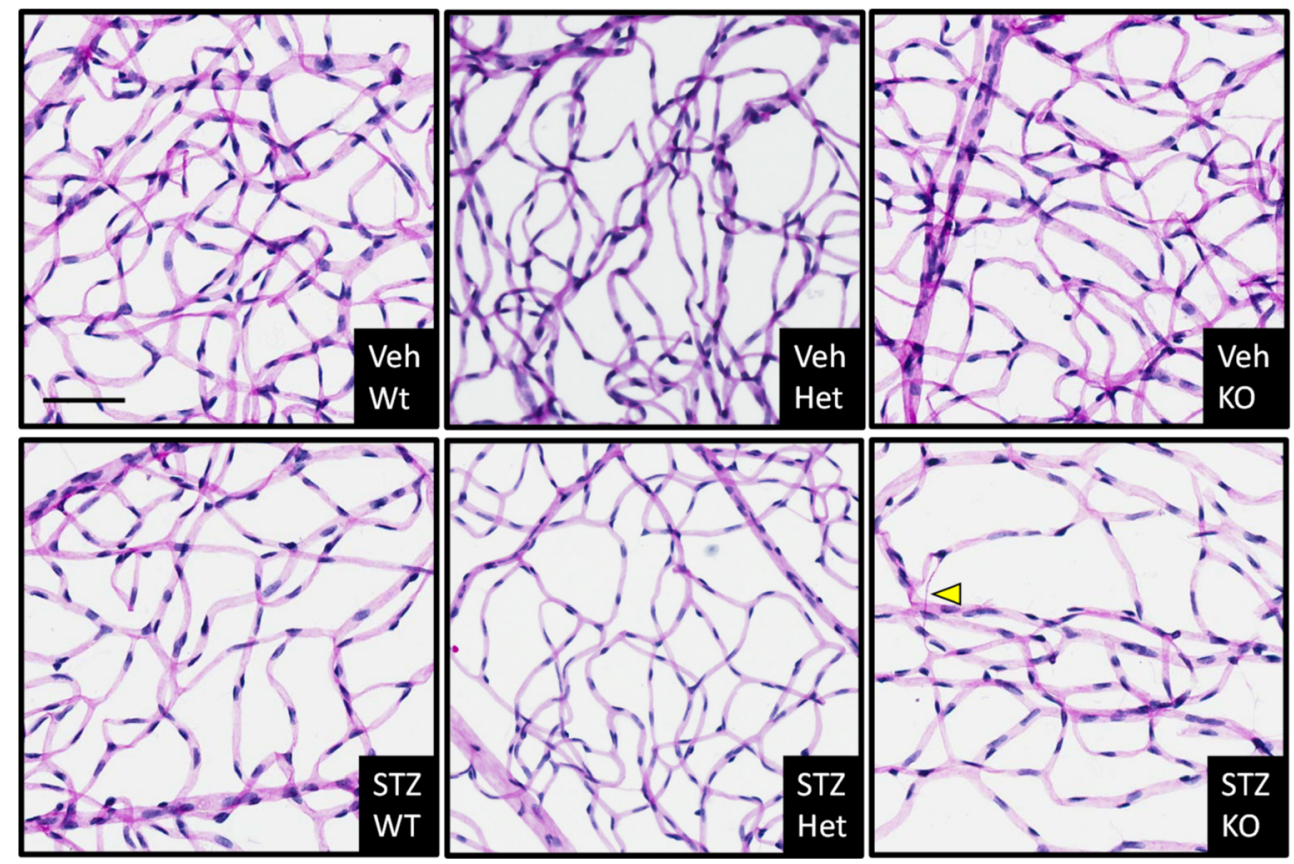